\title{
Control-source Electromagnetic Exploration and Open Access Journals
}

\section{Guo-qiang Xue*}

Institute of Geology and Geophysics, Chinese Academy of Sciences, Bei-Tu-Cheng-Xi-Lu 19, Chao-Yang District, Beijing, China

Controlled source audio magneto-telluric (CSAMT) method has received increasing attention for successful application as a subsurface imaging tool in mining, petroleum exploration, deep sea environmental investigations, groundwater studies, and geothermal exploration. Remarkable results were obtained by using CSAMT surveys to find fault and fissure-type water storage structures. The CSAMT method has also been extremely good at searching for certain ore structures, being able to delineate the abnormal shape and position of the body. In the prediction of geology in advance of mining, it is important to locate faults and karst caves. CSAMT has been successful in preventing and controlling disasters by detecting the distribution of water-bearing ore zones in the roof and floor ahead of extraction. Transient electromagnetic method (TEM) is a wide used geophysical exploration method which plays an increasingly important role in mineral exploration, environment and engineering studies, disaster forecasting, groundwater exploration, groundwater detection, underground shallow cavern location tunnel prediction. With the increased demand for a high resolution and an accurate imaging, it is necessary to further study the methodologies of the CSAMT and TEM so as to meet the requirement for fine structural interpretation. To meet this demand, worldwide researchers need to communicate and cooperate with each other in an efficient and effective way.

As seismic exploration proved to be the highest resolution in the imaging than those other geophysical methods, it is natural for us to introduce the main ideas of seismic processing methods into the TEM field. That is to convert the TEM field to the pseudo wave-field, and to use matured seismic data processing and interpreted techniques to the above pseudo wave-field. It is believed that the traditional TEM will be developed into multi-channel TEM method and become a hot research field in the future.

Because using the transient electromagnetic method (TEM) with the grounded electric source (GESM) can detect much deeper earth depth than that of loop source TEM, it is very important to study near zone TEM response due to grounded line source. It has become a kind of new developing orientation, so more and more researchers are trying to research in the near area mode. Measure electric fields in time domain has a potential advantage to sense deeper resistive targets because electric field measurements are more sensitive to charge buildup along the boundaries of electric resistivity when the vertical transient currents interacts with the horizontal layers.

For the calculations of transient electronic-magnetic (TEM) field, there are always some errors between the exact solutions and the approximate solution under dipole hypothesis. Electromagnetic field theory under the dipole hypothesis is the conventional and traditional, and has been widely accepted and applied for long time. Further, the theory has evolved into the point-dipole which is closed to pointcharge under macroeconomic significance. However, its fundament still is the dipole hypothesis and not the real point-charge, so the nature limitations of the dipole hypothesis still exist. Latest developments in this science field is based on the new theory of microwave antenna directly to use the point charge hypothesis on the microcosmic, and not the dipole approximation. The dipole hypothesis is replaced by the time-varying point charge, which can be matched to time-domain field. And this can provide the important inspiration for searching for the exact solution under the hypothesis of time-varying point charge so that the transient electromagnetic field in time domain can be directly solved.

Traditional academic journals play an important role in this regard in that they provide a platform for researchers to publish their problems and their solutions to problems. However, traditional academic journals are usually not open access publications, which limit their function as a communication platform for worldwide researchers. Therefore, new types of publications are needed, in particular in CSAMT and TEM.

Open access journals grant to all users a free, irrevocable, worldwide, and perpetual right of access to, and a license to copy, use, distribute, transmit and display the work publicly. With open access journals, worldwide researchers can publish their problems and their solutions to problems in a more flexible and interactive way. Now a new open access journal of OMICS Publishing Group, Journal of Geophysics \& Remote Sensing, has appeared. It will give authors to publish their newly research achievement and give reader a free way to benefit from OMICS Publishing Group's publications, especially Journal of Geophysics \& Remote Sensing.
*Corresponding author: Guo-qiang Xue, Institute of Geology and Geophysics, Chinese Academy of Sciences, Bei-Tu-Cheng-Xi-Lu 19, Chao-Yang District, Beijing, China, E-mail: ppxueguoqiang@163.com

Received March 16, 2012; Accepted March 16, 2012; Published March 20, 2012

Citation: Xue GQ (2012) Control-source Electromagnetic Exploration and Open Access Journals. J Geophys Remote Sensing 1:e102. doi:10.4172/21690049.1000e102

Copyright: (c) 2012 Xue GQ. This is an open-access article distributed under the terms of the Creative Commons Attribution License, which permits unrestricted use, distribution, and reproduction in any medium, provided the original author and source are credited. 